\title{
ESTUDO DA SÍNTESE DA ZEÓLITA ZSM-5 A PARTIR DE ARGILAS NATURAIS
}

Marcelo L. Mignoni, Chaline Detoni e Sibele B. C. Pergher*

Departamento de Química, Universidade Regional Integrada do Alto Uruguai e das Missões, Campus Erechim, Av. Sete de Setembro, 1621, 99700-000 Erechim - RS, Brasil

Recebido em 20/10/05; aceito em 11/4/06; publicado na web em 22/8/06

STUDY OF ZSM-5 ZEOLITE SYNTHESIS FROM NATURAL CLAYS. The synthesis of ZSM-5 zeolite was studied by three synthesis methods: standard synthesis, three day synthesis and synthesis employing clays (kaolin and montmorillonite). The raw materials and prepared materials were characterized by Chemical Analysis by X-Ray Fluorescence (XRF) and X-Ray Diffraction (XRD). The results show that it is possible to synthesized ZSM-5 zeolite using clays as raw materials. Kaolin gives phases of higher crystalinity than montmorillonite.

Keywords: ZSM-5; zeolites; clays.

\section{INTRODUÇÃO}

A zeólita ZSM-5 pertence a uma família de zeólitas designadas com as iniciais de sua inventora ZSM ("Zeolite Socony Mobil”), também conhecida como pentasil. Estas zeólitas se caracterizam por apresentarem um alto grau percentual de silício (relação $\mathrm{Si} / \mathrm{Al}$ compreendida entre 15 e infinito $)^{1}$.

Esta zeólita, em função desta relação $\mathrm{Si} / \mathrm{Al}$, apresenta os seguintes parâmetros de cela unitária: $\mathrm{a}=20,10-20,07 \AA ⿻$ § $\mathrm{b}=19,90$

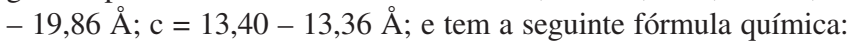
$x$ (TPA) $)_{2} \mathrm{O}-y \mathrm{Na}_{2} \mathrm{O}-z \mathrm{SiO}_{2}-h \mathrm{H}_{2} \mathrm{O}^{1}$.

A estrutura apresenta dois sistemas de canais que se entrecruzam, um que é retilíneo e o outro é sinosuidal, aos quais se ingressa por aberturas formadas por dez membros (10MR) de diâmetro cerca de $6 \AA^{2}$ (Figura 1).

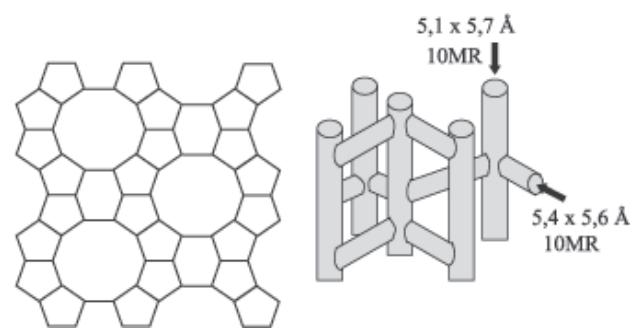

Figura 1. Representação estrutural da Zeólita ZSM-5²

A síntese da zeólita ZSM-5 é bem conhecida na literatura ${ }^{3-9}$. Na síntese destes materiais utilizam-se vários reagentes: sílica, aluminato de sódio, hidróxido de sódio, água, reagente orgânico (template) etc. A síntese é realizada em autoclaves com aquecimento e a cristalização da fase zeolítica leva de uma a duas semanas.

$\mathrm{Na}$ busca de novas rotas de sínteses de zeólita, é interessante obter as fases zeolíticas em menores tempos e empregando menores quantidades de reagentes orgânicos (viabilidade econômica).

O objetivo deste trabalho é estudar e propor novas rotas de síntese para a zeólita ZSM-5. Como referência empregaremos a síntese padrão ${ }^{1}$ e uma síntese proposta com o emprego de álcool como co-direcionador e em menor tempo de síntese ${ }^{10}$; posteriormente, será proposta uma síntese empregando argilas como fontes alternativas

*e-mail: pergher@uri.com.br de matérias-primas.

Estudou-se também a influência da agitação sobre o processo de cristalização dessa zeólita, uma vez que se conhece a influência deste parâmetro sobre a cristalização do gel ${ }^{1}$.

\section{PARTE EXPERIMENTAL}

A síntese da zeólita ZSM-5 foi realizada através de três procedimentos de síntese: síntese padrão ${ }^{1}$, síntese três dias ${ }^{10}$ e síntese empregando argilas.

\section{Síntese padrão}

A Tabela 1 mostra as relações molares dos reagentes empregados para a síntese da zeólita ZSM-5. A partir destas relações mola-

Tabela 1. Composição de gel e condições de síntese da zeólita ZSM5 (relações molares)

\begin{tabular}{|c|c|c|c|}
\hline \multicolumn{2}{|c|}{ Síntese padrão } & \multicolumn{2}{|c|}{ Síntese três dias } \\
\hline $\mathrm{H}_{2} \mathrm{O} / \mathrm{SiO}_{2}$ & 35,5 & $\mathrm{H}_{2} \mathrm{O} / \mathrm{SiO}_{2}$ & 24 \\
\hline $\mathrm{OH}^{2} / \mathrm{SiO}_{2}^{2}$ & 0,49 & $\mathrm{OH}^{2} / \mathrm{SiO}_{2}^{2}$ & 0,35 \\
\hline $\mathrm{Al}_{2} \mathrm{O}_{3} / \mathrm{SiO}_{2}^{2}$ & 0,02 & $\mathrm{Al}_{2} \mathrm{O}_{3} / \mathrm{SiO}_{2}^{2}$ & $1: 60$ \\
\hline $\mathrm{TPABr} / \mathrm{SiO}_{2}$ & 0,10 & $\mathrm{TPABr} / \mathrm{SiO}_{2}$ & 0,006 \\
\hline $\mathrm{Na} / \mathrm{SiO}_{2}$ & 0,49 & $\mathrm{Na} / \mathrm{SiO}_{2}$ & 0,35 \\
\hline $\mathrm{H}_{2} \mathrm{SO}_{4} / \mathrm{SiO}_{2}$ & 0,21 & Álcool/ $\mathrm{SiO}_{2}$ & 2 \\
\hline Temperatura & $95^{\circ} \mathrm{C}$ & Temperatura & $150^{\circ} \mathrm{C}$ \\
\hline Tempo & $9,11,13,14$ dias & Tempo & 3 dias \\
\hline
\end{tabular}

TPABr: Brometo de tetrapropilamônio

Tabela 2. Quantidades (g) dos reagentes empregados nas sínteses da zeólita ZSM-5

\begin{tabular}{llll}
\hline Síntese padrão $(\mathrm{g})$ & & Síntese três dias $(\mathrm{g})$ \\
\hline $\mathrm{SiO}_{2}$ & 18,0 & $\mathrm{SiO}_{2}$ & 9,0 \\
$\mathrm{NaOH}$ & 5,9 & $\mathrm{NaOH}$ & 2,064 \\
Aluminato de Sódio & 0,9 & Aluminato de Sódio & 0,455 \\
$\mathrm{TPABr} \mathrm{HO}_{4}$ & 8,0 & TPABr & 0,24 \\
$\mathrm{~S}_{2} \mathrm{AO}_{4}$ & 6,2 & Álcool & 13,8 \\
& 192 & Água & 64,8 \\
\hline
\end{tabular}

TPABr: Brometo de tetrapropilamônio 
res calcula-se a quantidade de cada reagente a ser empregada na síntese (Tabela 2).

Preparam-se três soluções: (A) diluindo a sílica em água; (B) dissolvendo aluminato de sódio e hidróxido de sódio em água; (C) dissolvendo o brometo de tetrapropilamônio (TPABr) em água, e posteriormente se adicionam cerca de 20 gotas de $\mathrm{H}_{2} \mathrm{SO}_{4} 98 \%$. As soluções $\mathrm{B}$ e C são simultaneamente misturadas sob agitação à solução A. O gel assim preparado é colocado em autoclaves de aço inoxidável com fundas internas de teflon ( $60 \mathrm{~mL}$ de capacidade). A temperatura de cristalização empregada é de $95^{\circ} \mathrm{C}$.

\section{Síntese três dias}

Este procedimento está baseado em uma patente ( $\mathrm{n}^{\circ}$ 8506248) registrada pela PETROBRÁS tendo como inventor Lam Yiu Lau ${ }^{10}$. As Tabelas 1 e 2 mostram as relações $\mathrm{Si} / \mathrm{Al}$ e as quantidades dos reagentes empregados nesta síntese, em comparação com a síntese padrão.

Prepara-se uma solução contendo $10 \mathrm{~g}$ de $\mathrm{H}_{2} \mathrm{O}$ e 2,064 g de $\mathrm{NaOH}$. Adiciona-se a esta solução $0,455 \mathrm{~g}$ de aluminato de sódio. Em seguida, adicionam-se 54,8 g de $\mathrm{H}_{2} \mathrm{O}$ e 0,24 g de TPABr. Como co-direcionador de estrutura emprega-se $13,8 \mathrm{~g}$ de álcool etílico. Finalmente, se adicionam $9,0 \mathrm{~g}$ de $\mathrm{SiO}_{2}$ e se mantém o gel sob agitação por $30 \mathrm{~min}$. O gel assim preparado é colocado em autoclaves de aço inoxidável com fundas internas de teflon $(60 \mathrm{~mL}$ de capacidade). A temperatura de cristalização empregada é de $150^{\circ} \mathrm{C}$.

\section{Síntese empregando argilas}

Neste procedimento emprega-se a composição do gel de síntese do procedimento padrão, a diferença se dá no emprego de duas argilas como fontes de Si e Al.

As argilas empregadas foram um caulim, denominado MINA, proveniente da Mineração Tabatinga Ltda. situada em Tijucas do Sul - PR, e uma montmonillonita ${ }^{11,12}$ proveniente da PETROSIX situada em São Mateus do Sul - PR.

\section{Caracterização dos materiais}

Os materiais preparados, bem como as argilas empregadas como matérias-primas, foram caracterizados por DRX e análises químicas. O equipamento utilizado para as análises de DRX foi um Diffraktometer modelo D5000 (Siemens) utilizando filtro de Ni e radiação $\mathrm{Cu}-\mathrm{k} \alpha(\lambda=1,54 \AA)$. As análises químicas foram realizadas por fluorescência de raios X (FRX). O equipamento empregado foi um Philips modelo PW 1480.

\section{RESULTADOS E DISCUSSÃO}

A Figura 2 ilustra os difratogramas dos sólidos obtidos pela síntese padrão. Comparando os picos obtidos com os da ZSM-5 padrão da literatura ${ }^{13}$, observa-se que a fase zeolítica presente é a ZSM-5. Os picos principais estão assinalados e descritos na Tabela 3. Observa-se que em 9 dias de síntese a fase zeolítica ZSM-5 está presente, entretanto com baixa cristalinidade. Com o aumento do tempo de síntese aumenta a cristalinidade dos materiais obtidos. Chega-se a conclusão que é requerido pelo menos 14 dias de síntese para obter-se a ZSM-5 pura e cristalina.

A síntese proposta (3 dias) emprega álcool como co-direcionador de estrututa e utiliza ácido sulfúrico em quantidade inferior, além de diminuir consideravelmente a quantidade de template (TPABr), o que torna o procedimento mais viável do ponto de vista econômico. Após 3 dias de síntese, os materiais obtidos apresentam os difratogramas ilustrados na Figura 3. Observa-se que os materiais

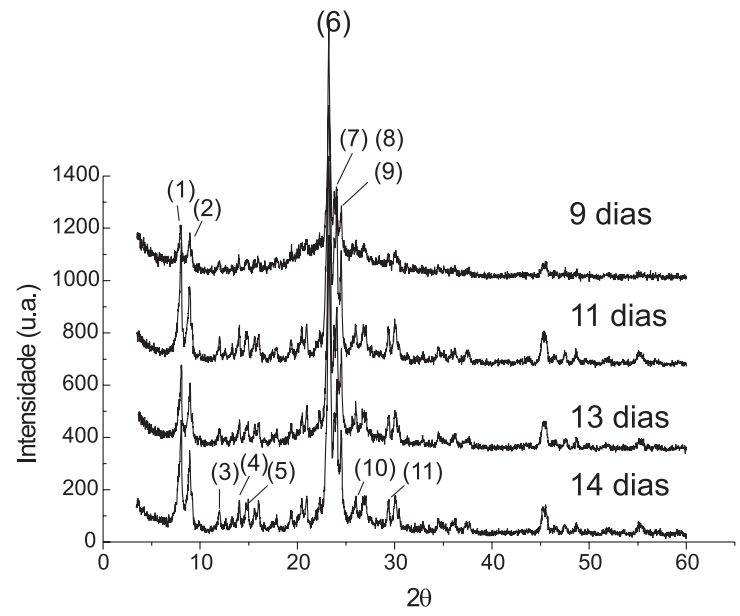

Figura 2. Difratogramas de raios- $X$ dos materiais obtidos pela síntese padrão

Tabela 3. Posições e intensidades relativas dos picos de difração obtidos nos materiais preparados pela síntese padrão da ZSM-5

\begin{tabular}{lcc}
\hline $2 \theta$ & I relativa & Picos* \\
\hline 7,95 & 63,4 & 1 \\
7,96 & 46,7 & 1 \\
8,83 & 29,1 & 2 \\
8,89 & 34,1 & 2 \\
9,11 & 23,6 & 2 \\
11,93 & 9,9 & 3 \\
13,95 & 16,2 & 4 \\
14,83 & 10,1 & 5 \\
23,19 & 100,0 & 6 \\
23,32 & 78,5 & 6 \\
23,74 & 31,1 & 7 \\
23,99 & 48,2 & 8 \\
24,46 & 40,3 & 9 \\
25,97 & 13,8 & 10 \\
29,34 & 12,1 & 11 \\
\hline
\end{tabular}

* picos assinalados na Figura 2

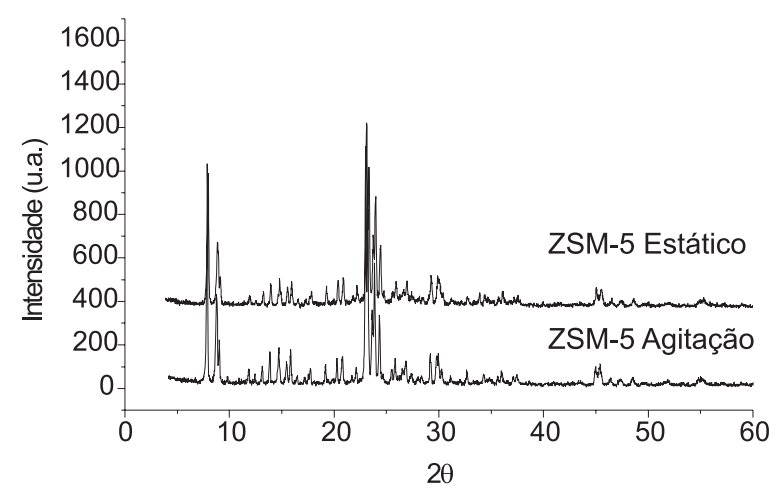

Figura 3. Difratogramas de raios-X dos materiais obtidos pela síntese três dias

são altamente cristalinos e que a fase zeolítica obtida é a ZSM-5 pura. Conclui-se que, com a síntese proposta, se pode obter a ZSM5 de elevada cristalinidade em apenas 3 dias, empregando quantidades menores de template.

Uma vez que estes resultados foram bastante satisfatórios, resolveu-se empregar a metodologia anterior de síntese para preparar a Zeólita ZSM-5 utilizando argilas como nova fonte de Si/Al.

A Tabela 4 apresenta os resultados de caracterização da maté- 
Tabela 4. Caracterização da argila Mina

\begin{tabular}{lcll}
\hline Análise química & \multicolumn{3}{l}{ Análise mineralógica } \\
\hline $\mathrm{SiO}_{2}(\%)$ & 54,45 & Caulinita (\%) & 79 \\
$\mathrm{Al}_{2} \mathrm{O}_{3}(\%)$ & 34,33 & Mica (\%) & 4 \\
$\mathrm{Fe}_{2} \mathrm{O}_{3}(\%)$ & 1,43 & Quartzo (\%) & 12 \\
$\mathrm{MgO}(\%)$ & 0,09 & Gibsita (\%) & 5 \\
$\mathrm{MnO}(\%)$ & 0,01 & & \\
$\mathrm{CaO}(\%)$ & 0,09 & & \\
$\mathrm{~K}_{2} \mathrm{O}(\%)$ & 0,21 & & \\
$\mathrm{Na}_{2} \mathrm{O}(\%)$ & 0,05 & & \\
$\mathrm{P}_{2} \mathrm{O}_{5}(\%)$ & 0,04 & \\
$\mathrm{Zr}(\mathrm{mg} / \mathrm{L})$ & 785 & & \\
\hline
\end{tabular}

ria-prima MINA. Observa-se que a mesma é essencialmente uma caulinita e que possui alto teor de $\mathrm{Si}$ e $\mathrm{Al}$, sendo então indicada para a síntese de zeólitas. A argila também possui pequenas quantidades de vários metais compensadores de carga, que poderão influenciar no processo de síntese.

O difratograma de Raios X da argila natural está representado na Figura 4, onde se observa que o material é constituído de caulinita, mica, gibsita e quartzo. A caulinita possui estrutura lamelar formada pelo empilhamento regular de camadas 1:1 onde cada camada consiste de uma folha de tetraedros de Si e uma folha de octaedros de $\mathrm{Al}$, com espaçamento basal de aproximadamente 7,0 $\AA$ (Figura 5).

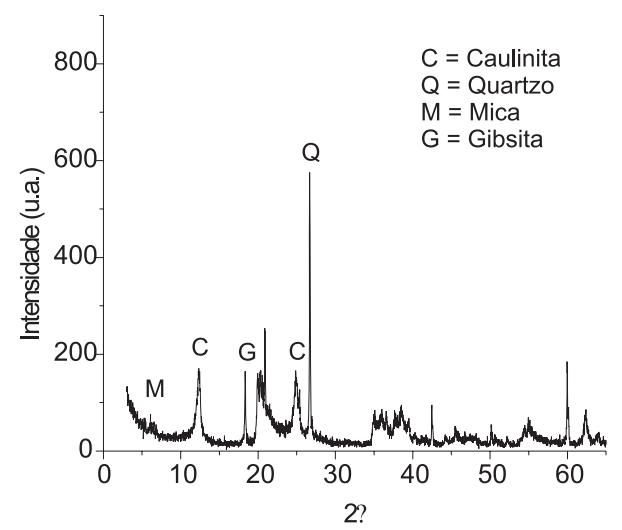

Figura 4. Difratograma de raios- $X$ da argila natural Mina

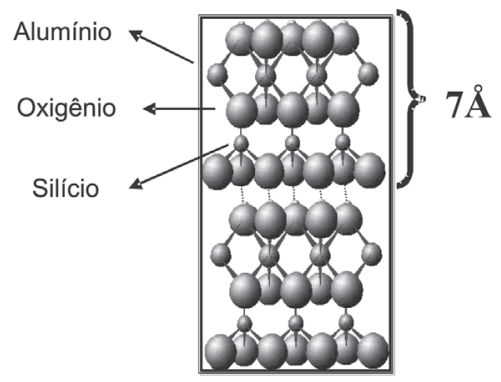

Figura 5. Estrutura da caulinita

A Tabela 5 apresenta os resultados de caracterização da matéria-prima montmorillonita que se caracteriza essencialmente por ser uma esmectita de alto teor de Si e Al. Por outro lado, nota-se que possui pequenas quantidades de vários metais compensadores de carga que poderão influenciar no processo de síntese.

A Figura 6 apresenta o resultado de DRX da argila montmorillonita, confirmando que se trata do material lamelar com estrutura (2:1), ou
Tabela 5. Análise química da argila montmorillonita

\begin{tabular}{lc}
\hline Óxidos & $(\%)$ \\
\hline $\mathrm{Al}_{2} \mathrm{O}_{3}$ & 23,01 \\
$\mathrm{SiO}_{2}$ & 60,86 \\
$\mathrm{CaO}$ & 2,29 \\
$\mathrm{Fe}_{2} \mathrm{O}_{3}$ & 5,60 \\
$\mathrm{~K}_{2} \mathrm{O}$ & 1,10 \\
$\mathrm{SO}_{3}$ & 0,2 \\
$\mathrm{MgO}_{\mathrm{Mn}}$ & 6,49 \\
$\mathrm{TiO}_{3}$ & 0,11 \\
$\mathrm{P}_{2} \mathrm{O}_{5}$ & 0,29 \\
\hline
\end{tabular}

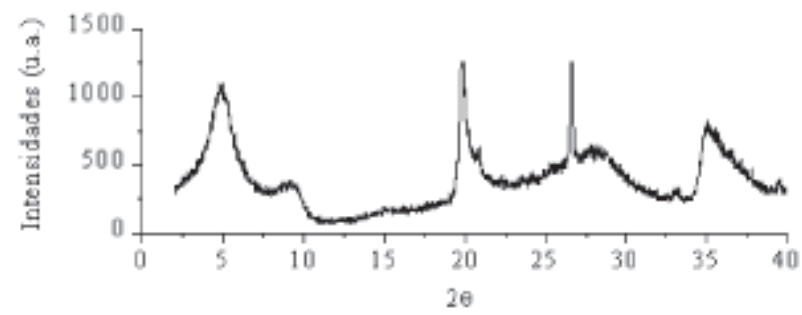

Figura 6. Difratograma de raios- $X$ da argila natural montmorillonita

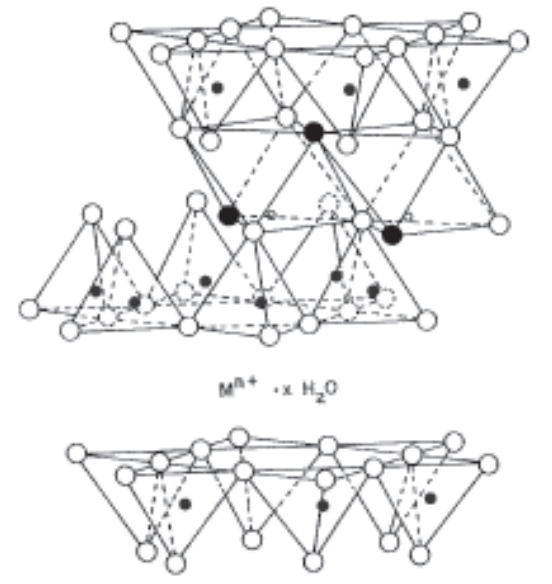

$\mathrm{SiO}_{4}$

$\mathrm{AlO}_{6}$

$\mathrm{SiO}_{4}$

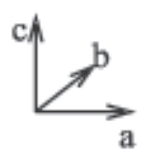

Figura 7. Estrutura idealizada de uma esmectita (2:1). O - átomos de oxigênio; - grupos -OH; $\bullet$ - átomo de silício $e^{\circ}$ - átomo de alumínio

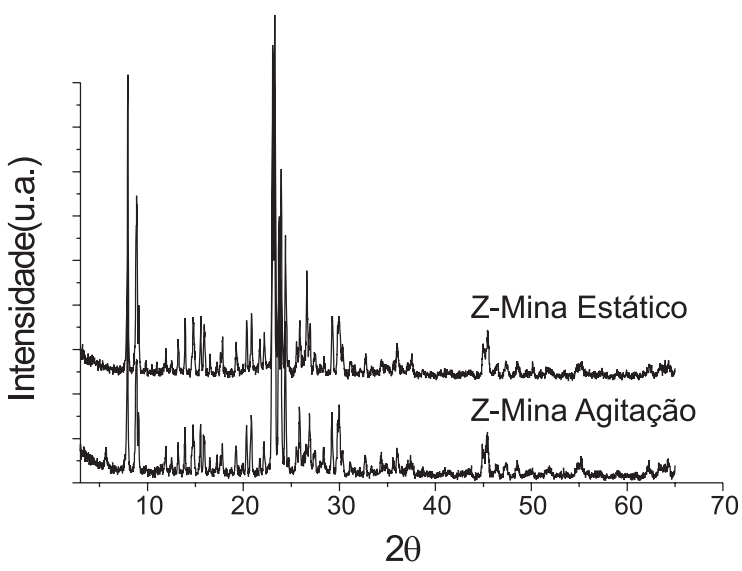

Figura 8. Difratogramas de raios-X utilizando argila Mina 


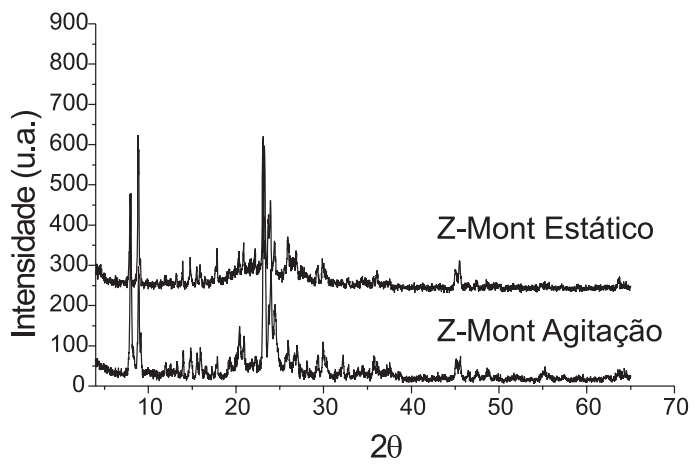

Figura 9. Difratogramas de raios-X utilizando argila montmorillonita

seja, duas lamelas de silício e uma de alumínio (Figura 7).

Os materiais obtidos empregando ambas argilas foram analisados por difração de Raios X. Comparando estes resultados (Figura 8 e 9) com os anteriores (Figuras 2 e 3), observa-se que a fase zeolítica presente é a ZSM-5. Nota-se que para a argila Mina a cristalinidade é maior, quando comparada com a cristalinidade obtida para a montmorillonita (Tabela 6). $\mathrm{O}$ processo em agitação favorece a formação de fases zeolíticas conduzindo a fases mais cristalinas que as amostras sintetizadas em estático.

Tabela 6. Cristalinidade* do material ZSM-5 (\%)

\begin{tabular}{ll}
\hline Z-Mina agitação & 100 \\
Z-Mina estático & 88 \\
Z-Mont agitação & 65,5 \\
Z-Mont estático & 42,3
\end{tabular}

*Cristalinidade calculada usando a razão da soma das áreas dos 3 picos mais intensos das amostras preparadas $(2 \theta=22,6 ; 23,2 ; 24,6)$ multiplicados por 100 .

As argilas foram empregadas na sua forma natural; em trabalhos da literatura ${ }^{14-16}$ constata-se que normalmente se requer que o caulim seja previamente calcinado transformando-se em metacaulim. Acredita-se que na forma meta-caulim se encontre mais reativo e, por isso, mais adequado para a síntese de zeólitas.

$\mathrm{Na}$ literaura não se relata o emprego de argilas montmorillonitas. Observa-se com este estudo que é possível sintetizar a zeólita ZSM5 empregando argilas como fonte de $\mathrm{Si}$ e $\mathrm{Al}$, sendo que, mesmo na forma natural (sem necessidade de calcinação), obtêm-se fases zeolíticas. Os resultados mostram que a caulinita conduz a fases mais cristalinas que a montmorillonita. Uma explicação para este fato seria que o caulim possui 2 camadas (1:1), enquanto que a montmorillonita possui 3 camadas (2:1), sendo mais difícil sua transformação em zeólita.

\section{CONCLUSÕES}

É possível sintetizar a zeólita ZSM-5 empregando argilas como fontes de silício e alumínio. Especificamente a caulinita conduz a fases ZSM-5 mais cristalinas que a montmorillonita, e mesmo na forma natural (sem calcinação) se obtêm fases zeolíticas.

\section{AGRADECIMENTOS}

À FAPERGS, URI - Campus Erechim e ao CNPq pelo auxílio financeiro e ao Laboratório de Difração de raios-X - UFRGS pelas análises de DRX.

\section{REFERÊNCIAS}

1. Gianeto, G. P.; Zeolitas Características, propriedades y aplicaciones industriales, Ediciones Innovación Tecnológica: Caracas, 1990.

2. Pergher, S. B. C.; Oliveira, L. C. A.; Smaniotto, A.; Petkowicz, D. I.; Quim. Nova 2005, 28, 751

3. Cardoso, D.; Carvalho, C. C.; Silva, M. A. P.; Cardoso, M. J. B.; $7^{\circ}$ Seminário Brasileiro de Catálise, Gramado, Brasil, 1993.

4. Valencia, S. V.; Tese de Doutorado, Universidade Politécnica de Valencia, Espanha, 1997.

5. Smart, L.; Moore, E.; Química del estado sólido, una introducción, Ed. Addison- Wesley Iberoamericana: Espanha, 1995, cap. 5

6. Oliveira, K. D.; Muniz, L. R.; Zeolitas: síntese e fabricação, Departamento de Engenharia Química da EPUSP: São Paulo, 1989.

7. Luz, A. B.; Zeólitas: propriedades e usos industriais, Série Tecnologia Mineral, CETEM/CNPq: Rio de Janeiro, 1995, p. 68.

8. Bosch, P.; Schifter, I.; Fondo De Cultura Económica, La ciencia para Todos, México, 1997.

9. Minatelli, D. C.; Dissertação de Mestrado, Universidade de São Paulo, Brasil, 1992.

10. Lau, L. Y.; BR PI 8506248, 1987.

11. Pergher, S. B. C.; Sprung, R.; Quim. Nova 2005, 28, 777.

12. Pergher, S. B. C.; Dissertação de Mestrado, Universidade Estadual de Maringá, Brasil, 1993.

13. Ballmoos, R. E.; Higgins, J. B.; Collection of Simulated XDR Powder Patterns from zeolites, 2 ed., IZA: Amsterdam, 1990.

14. Basaldella, E. I.; Sánchez, R. T.; Vargas, S. L. P.; XVI Simpósio Iberoamericano de Catálisis, Cartagena de Indias, Colombia, 1998.

15. Abdmeziem, K. E.; Siffert, B.; Appl. Clay Sci. 1994, 437.

16. Contreras, Y.; Imbert, F.; Uzcátegui, A.; Delgado, G.; XVII Simpósio Iberoamericano de Catálise, Porto, Portugal, 2000. 